\title{
Conductas agresivas y de Bullying desde la perspectiva de actividad física, lugar de residencia y género en adolescentes de Granada
}

\section{Aggressive and bullying from the perspective of, physical activity, place of residence and sex behavior among adolescents in Granada}

Félix ZURITA ORTEGA, José Ma VILCHES AZNAR, Rosario PADIAL RUZ, Antonio J. PEREZ CORTES y Asunción MARTÍNEZ MARTÍNEZ Universidad de Granada

Recibido: Enero 2014

Aceptado: Marzo 2014

\section{Resumen}

En este trabajo de investigación se tiene como objetivo analizar y establecer los índices de Victimización, Bullying y Conducta Violenta y su posible relación con el género, residencia habitual y práctica de A.F de adolescentes de 13 a 17 años de Granada (España).

La participación de un total de 2.273 adolescentes permitió el registro y evaluación de las variables Victimización (escala de Victimización en la Escuela), Conducta Violenta (medida con la escala de Conducta Violenta en la Escuela), Bullying, género, lugar de residencia y práctica de Actividad Física.

Los resultados mostraron que los adolescentes en más de la mitad de los casos indicaron que sin ser agresores sí que fueron testigos de casos de Bullying, también se detectó que los niveles de Agresividad (Manifiesta y Relacional) eran similares, mientras que la Victimización de tipo Verbal era la más usual. Asimismo los jóvenes residentes fuera del contexto familiar eran más acosadores y habían sufrido más acoso y tenían mayores índices de agresividad y victimización. La práctica de Actividad Física era más asidua en varones, y estos se caracterizaban por tener mayor agresividad manifiesta y un índice superior de victimización física, encontrándose más acosadores entre los varones y siendo estos más testigos de hechos violentos, mientras las chicas lo sufrían más y a la vez percibían menos situaciones de Bullying.

Como principal recomendación indicamos la necesidad de estudiar con mayor profundidad a la población deportivamente activa para intentar dilucidar la casuística de estos resultados.

Palabras clave: agresividad, deporte, adolescente, distribución por sexo, colegio internado.

\section{Abstract}

In this research the aim was to analyze and establish the rates of victimization, bullying and violent conduct and its possible relationship to gender, habitual residence and Physical Activity Practice among teens from13-17 years of Granada (Spain).

The participation of a total of 2,273 adolescents allowed the registration and evaluation of 
Victimization (Scale Victimization at School), Violent Conduct (measured with the scale of violent conduct at the School), Bullying, Gender, Place of residence and Physical Activity practice variables.

The results showed that adolescents in over half of the cases indicated that without being perpetrators themselves had witnessed cases of bullying, also found that levels of aggressiveness (Manifest and Relational) were similar, while the verbal victimization was more usual. It was also found that young teenagers residing outside the family context were bullies and had suffered further harassment and had higher rates of aggressiveness and victimization.

The practice of physical activity was more frequent in males, and these were characterized by greater manifestation of aggressiveness and a higher rate of physical victimization, existing more stalkers among men and still more witnesses to these violent events, while the girls suffered more and perceived less bullying situations. It could be indicated as a main recommendation the need to further explore the physically active population to try to elucidate the casuistry of these results.

Keywords: aggressiveness, sport, adolescents, sex distribution, boarding school.

Desde los primeros estudios realizados por Olweus (1978) con adolescentes, las cifras de incidencia de violencia escolar, "Bullying", ha aumentado de una forma preocupante en todo el mundo (Smith, 2003; Cava, Musitu y Murgui, 2007; Cook, Williams, Guerra, Kim y Sadek, 2010; Yubero, Ovejero y Larrañaga, 2010; Povedano, Estévez, Martínez y Monreal, 2012; Pechorro, Goncalves, Maroco, Gama, Neves y Nunes, 2014; Zeigler-Hill, Enjaian, Holden y Southard, 2014). Los estudios de investigación realizados en España apuntan a que su incidencia va incrementándose paulatinamente. El Informe del Defensor del Pueblo, en su primera edición en 2000 apuntaba que el Bullying se aprecia en más del 50\% de los centros escolares y en más de la mitad de los alumnos e incluso se hacen afirmaciones sobre la edad donde el ser humano es más vulnerable (Martorell, González, Ordoñez y Gómez, 2011).

En sus estudios Oppenheimer (2008) y Arias (2013), señalan que la violencia se va incrementando en las diversas sociedades mundiales, refiriéndose principalmente a Estados Unidos y América Latina, donde señalan más de 5,4 millones de crímenes violentos frente a los 115.000 de España, lo que lo sitúa entre los países con menos crímenes pero indicando que va a aumentándose de forma paulatina la violencia. Respecto al concepto de violencia escolar existe cierta homogeneidad y consenso en considerarla como aquella conducta intencionada mediante la cual se causa un daño a otra persona dentro del centro educativo o en cualquier otra actividad organizada por éste, pudiendo ser ejercida o padecida por cualquier miembro de la comunidad educativa (Guerra, Álvarez-García, Dobarro, Núñez, Castro y Vargas, 2011), al hilo de esto Blaya (2010) señala que las víctimas no solamente interaccionan en el centro escolar sino que también se ven afectadas fuera de él, siendo el ciberbullying otro elemento a considerar.

En este sentido, la mayor parte de la investigación en este ámbito centra su interés en el estudio de los denominados agresores y víctimas (Choe y Zimmerman, 2014; Haynie, Doogan y Soller, 2014; Pabayo, Molnar y Kawachi, 2014). Sin embargo, algunos autores señalan que ambas categorías no son mutuamente excluyentes, ya que 
al menos la mitad de las víctimas manifiestan también agredir a otros compañeros (Austin y Joseph, 1996). Las víctimas, generalmente, se caracterizan por sus conductas pasivas y sumisas, mientras que los agresores/víctimas tienden a mostrar conductas hostiles, en muchas ocasiones como respuesta a la victimización que sufren (Schwartz, 2000 y Estévez, Inglés, Emler, Martínez-Monteagudo y Torregrosa, 2012).

En cuanto al tipo de conducta violenta, los escolares objeto de estudio en diferentes investigaciones, señalan como más frecuente la violencia verbal (insultos, amenazas, intimidación, descalificaciones), seguida del aislamiento social. Tras la violencia verbal y la exclusión social, los comportamientos más prevalentes son las agresiones físicas directas y el presionar u obligar a otros a la comisión de agresiones (pegar, chantajear), y por último, estarían las amenazas con armas y el acoso sexual. (Rodríguez, Seasone y Pedreira, 2006 y Defensor del Pueblo 2007). En la actualidad ha surgido un nuevo modus operandi de la formas de violencia escolar denominada cyberbullying (Oliveros y Barrientos, 2007; Ortega, Calmaestra y Mora, 2008 y Cerezo, 2009).

Para determinar los comportamientos violentos debemos seguir las directrices de Little, Henrich, Jones y Hawley (2003), que los han clasificado según la función de su naturaleza o forma de manifestación como violencia física, verbal y relacional.

Teniendo en cuenta que estamos ante un problema de doble sentido: agresión versus victimización, se pueden establecer comparaciones entre los elementos que facilitan uno u otro comportamiento (Cerezo, 2009). Entre los principales elementos Carroll, Houghton y Baglioni (2000); Cerezo y Ato (2010); López-Romero y Romero (2010) o Buelga, Cava y Musitu (2012) nos explicitan el término de reconocimiento social de los agresores que desencadena en una reputación o imagen social determinada frente al resto de compañeros que puede determinar el grado de integración o rechazo del individuo en un grupo o comunidad, por tanto ya que la reputación social juega un papel estelar en la vida de los jóvenes, los estudiantes tratan de conseguir una imagen social no conformista en diferentes contextos, como son la escuela o el barrio donde viven. De hecho, algunos adolescentes se implican en conductas violentas o delictivas en sus comunidades para tratar de obtener un reconocimiento social como popular o rebelde (Buelga et al., 2012) y en el caso de los varones en ocasiones se sustentan en la teoría de la masculinidad hegemónica (Connell, 1995), si bien el desvío de esta masculinidad como señala Renold (2004) implica actitudes marginadas.

Los estudios sobre género constituyen un contexto multi y transdisciplinar, iniciándose su estudio en la década de los 80 (Scott, 1992), estos trabajos versan primordialmente sobre el conocimiento de las relaciones de índole sociocultural entre hombres y mujeres (González- Jiménez, 2009).

Partiendo de lo planteado anteriormente, diversos autores como Abarca, Carvajal y Cifuentes (2012) nos señalan que dentro de los estudios actuales sobre masculinidad, se está provocando una crisis transformacional provocándose un cambio en el modelo hegemónico planteado por Connell (1995), modificándose el esquema que culturalmente se encontraba asociado al hombre con elementos atributivos de independencia, agresividad y conductas violentas. 
Frente a la aseveración anterior la participación de los adolescentes en actividades formales o informales de su comunidad, está asociada con un mejor ajuste psicológico y social (Trickett, 2009). De este modo Pederson, Seidman, Yoshikawa, Rivera y Allen (2005) afirman que la alta implicación de los adolescentes en contextos comunitarios se relaciona con una menor participación en actos delictivos o violentos. Cualquier aproximación explicativa hacía el Bullying debe puntualizar que aunque el componente de personalidad es muy importante, no lo es menos el contexto social (Cerezo, 2009), así incluso cabe la posibilidad de ver de dónde emana la problemática, ya sea del entorno familiar (Lazo y Salazar, 2011), escolar o de actividades realizadas.

En nuestro caso uno de los elementos que considerábamos importante dada la escasez de estudios que comparasen a adolescentes residentes en domicilio familiar y fuera de él, era constatar si los alumnos en régimen de internado (no vivían con sus padres) poseían niveles de agresividad hacía sus compañeros similares a los que si residían en el domicilio familiar, por tanto en relación a este último aspecto, en el presente estudio nos hemos planteado los siguientes objetivos:

- Analizar el nivel de Victimización, Bullying, Conducta Violenta y Variables Familiares-Deportivas en adolescentes de 13 a 17 años.

- Comprobar la correspondencia existente entre parámetros de género, residencia y actividad física con comportamientos de Victimización, Agresividad y Bullying.

- Establecer la relación entre Victimización, Conducta Violenta y Bullying.

Este estudio nos reporta datos novedosos sobre el perfil de adolescentes ya presenten signos de victimización o agresividad y la posible relación que tiene con el lugar de residencia habitual, género o práctica continua de actividad físico deportiva.

\section{Material y Método}

\section{Participantes}

Participaron en esta investigación de carácter descriptivo y de tipo transversal un total de 2.273 adolescentes españoles, de ambos sexos $(53,1 \%$ de chicos y $46,9 \%$ de chicas), de 13 a 17 años ( $M=14,94$ años; $\mathrm{DT}=1,258)$, de 13 centros escolares públicos $(\mathrm{N}=6)$ y concertados/privados $(\mathrm{N}=7)$, ubicados en distintas zonas de la ciudad de Granada y distribuidos en los cuatro cursos de la Educación Secundaria Obligatoria (ESO) y residencias escolares. La muestra es representativa de la comunidad educativa donde se desarrolla el estudio que presenta un universo poblacional de 11,030 alumnos de ESO, asumiendo un error muestral de 0,02 , con un nivel de confianza del $95 \%$, por lo que la muestra requerida fue de 1.972 adolescentes. Para seleccionar los participantes se establece un muestreo por conglomerados como indican Santos, Muñoz, Juez y Cortiñas (2003).

Las unidades de muestreo empleadas son los centros educativos y residencias escolares de la ciudad de Granada (cuatro residencias internados). Los estratos vienen establecidos por el lugar de residencia habitual (hogar familiar o residencia), por lo que 
siguiendo el propósito de este trabajo la muestra queda dividida en dos categorías: los que viven en domicilio familiar $(\mathrm{N}=2.070 ; 91,1 \%)$ y los que residen en centros y residencias internados escolares $(\mathrm{N}=203 ; 8,9 \%)$.

\section{Procedimiento}

En primer lugar, a través de la Facultad de Ciencias de la Educación de la Universidad de Granada (Área de Corporal), y en contacto con la Delegación de Educación de la Junta de Andalucía, se solicitó la colaboración de los Centros Educativos de la Capital de Granada seleccionados, a partir de un muestreo de conveniencia de las categorías objeto de estudio. La dirección de cada centro educativo fue informada sobre la naturaleza de la investigación y solicitando la colaboración de sus alumnos/as. En segundo lugar se adjuntó un modelo de autorización destinado a los responsables legales de los adolescentes pidiéndoles su consentimiento informado.

En todo momento se garantizó a los participantes el anonimato de la información recogida aclarando que su utilización sería sólo con fines científicos. Los encuestadores estuvieron presentes durante la recogida de los datos que se llevó a cabo en sus aulas habituales del centro escolar durante su periodo regular de clase, esta se desarrolló sin ningún tipo de problema o anormalidad a reseñar. En último lugar se agradeció a los docentes, orientadores y responsables su colaboración y se les informó del envío en un futuro próximo de un informe sobre los datos obtenidos respetando la confidencialidad de los mismos.

El estudio cumplió con las normas éticas del Comité de Investigación y Declaración de Helsinki de 1975. Se contó en todos los casos con el consentimiento informado de los participantes y se respetó el resguardo a la confidencialidad.

\section{Variables e instrumentos}

La presente investigación tomó como referencia las siguientes variables e instrumentos de medida, es decir:

- Género, según fuese masculino o femenino recogido mediante Ad-Hoc.

- Residencia, si el adolescente habitaba en el domicilio familiar o fuera de él, igualmente recogido mediante Ad-Hoc.

- Conducta Violenta en la Escuela, dividida en dos categorías Agresión Manifiesta o Directa o Agresión Relacional o Indirecta, subdividida a su vez cada una de ellas en tres subescalas (Pura, Reactiva o Instrumental), está propuesta en su versión original por Little et al., (2003) y adaptada por el Grupo Lisis (Estévez, 2005) y empleada en estudios de similares características entre otros por Cava, Musitu y Murguy (2006); Musitu, Estévez y Emler (2007) o Jiménez, Moreno, Murguy y Musitu (2008), se compone de una escala tipo Likert de 25 ítems que oscila entre los valores 1 (nunca) al 4 (siempre), que una vez puntuados nos dan dos tipos de conducta violenta: Agresión Manifiesta o Directa (la cual se genera en un encuentro cara a cara donde el agresor es identificable por la victima) o Agresión Relacional o 
Indirecta (considerada cuando el agresor permanece de una forma anónima). Presentan un coeficiente de fiabilidad Alpha de Cronbach de .824 para los ítems que miden la agresividad manifiesta y .722 para las cuestiones de la agresividad relacional, muy similares al .088 y .081, para ambas subescalas obtenido por Musitu, Estévez y Emler (2007).

- Escala de Victimización en la Escuela, dividida en tres subescalas, está extraído de la versión original de Mynard y Joseph (2000) y adaptada al español por Cava et al. (2007) y Jiménez, Musitu, Ramos y Murgui (2009), donde mediante una escala Liker de cuatro opciones $(1=$ Nunca a $4=$ Muchas veces), se valoran 20 ítems, que generan tres tipos de victimización en la escuela (Relacional, Física y Verbal). La consistencia interna (Alpha de Cronbach) del cuestionario es de $.924,(\mathrm{p}=.888$ para Relacional, $\mathrm{p}=.568$ para Física y $\mathrm{p}=.846$ Verbal), datos similares a los hallados en el estudio de Povedano et al., (2012).

- Actividad Física, con la opción de si práctica o no, utilizándose una hoja de registro para ello, donde se instaba a marcar la opción de $\mathrm{Si}$, siempre y cuando el adolescente realizase Actividad Física fuera del Centro Escolar con una frecuencia igual o superior a tres horas semanales y No, si no lo realizaba.

- Bullying, con cuatro opciones, según sea acosador, victima (sufrí acoso), no involucrado pero testigo y no testigo, no víctima y no acosador, empleándose para su recogida una hoja de registro con ítems concernientes a este parámetro.

\section{Análisis de Datos}

Para el análisis de los datos se utilizó el programa estadístico SPSS 20.0., en primer lugar se utilizó el alpha de Cronbach para establecer la consistencia interna de los ítems de los instrumentos empleados, a continuación se estableció la descripción de todas las variables presentes en el estudio (Agresividad, Victimización, Bullying, Actividad Física, Genero y Lugar de Residencia) mediante la utilización de desviación típica y medias, y para terminar el análisis se determinó el análisis comparativo empleando para ello las tablas de contingencia y ANOVA (Conducta Violenta y Victimización), para establecer las diferencias estadísticas entre las variables objeto del estudio

\section{Resultados}

Como puede observarse en la Tabla I, referente a los descriptivos de la muestra analizada, se constató que $1.208(53,1 \%)$ adolescentes eran varones y $1.065(46,9 \%)$ eran féminas. La mayor parte de ellos residían en el domicilio familiar $(91,1 \%$; $\mathrm{n}=2070$ ), y únicamente 203 adolescentes tenían su domicilio en residencia $(8,9 \%)$, asimismo $1.823(80,2 \%)$ practicaban actividad física de forma habitual al menos tres horas semanales fuera del contexto escolar frente al restante 19,8\% $(\mathrm{n}=450)$, asimismo el 52,1\% ( $\mathrm{n}=1.185)$ fueron testigos de casos de Bullying sin estar involucrados y únicamente un $5 \%(\mathrm{n}=114)$ señalaron ser acosadores. En lo que respecta a la conducta 
violenta se mostraron valores similares entre Agresividad Manifiesta $(M=1,29$; $\mathrm{DT}=0,55)$ y Relacional $(\mathrm{M}=1,26 ; \mathrm{DT}=0,51)$ y en victimización en la escuela se reportaron valores medios de $1,72(\mathrm{DT}=0,55)$ en Victimización Verbal, 1,53 (DT= $0,45)$ para Relacional y $1,27(0,34)$ para Victimización Física.

\begin{tabular}{|c|c|}
\hline \multicolumn{2}{|c|}{ Género } \\
\hline Masculino & $53,1 \%(\mathrm{n}=1.208)$ \\
\hline Femenino & $46,9 \%(n=1.065)$ \\
\hline \multicolumn{2}{|c|}{ Residencia Habitual } \\
\hline Domicilio Familiar & $91,1 \%(\mathrm{n}=2.070)$ \\
\hline No Domicilio familiar & $8,9 \%(n=203)$ \\
\hline \multicolumn{2}{|c|}{ Práctica de A.F } \\
\hline Si & $80,2 \%(\mathrm{n}=1.823)$ \\
\hline No & $19,8 \%(\mathrm{n}=450)$ \\
\hline \multicolumn{2}{|c|}{ Bullying } \\
\hline Testigo, no involucrado & $52,1 \%(\mathrm{n}=1.185)$ \\
\hline Acosador & $5,0 \%(\mathrm{n}=114)$ \\
\hline Víctima & $13,9 \%(n=315)$ \\
\hline Ninguna de las anteriores & $29,0 \%(n=659)$ \\
\hline \multicolumn{2}{|c|}{$\begin{array}{c}\text { Conducta Violenta } \\
\end{array}$} \\
\hline Agresividad Manifiesta & $\mathrm{M}=1,29(\mathrm{D} . \mathrm{T}=0,55)$ \\
\hline Agresividad Relacional & $\mathrm{M}=1,26(\mathrm{D} \cdot \mathrm{T}=0,51)$ \\
\hline \multicolumn{2}{|c|}{ Victimización } \\
\hline Victimización Relacional & $\mathrm{M}=1,53$ (D.T $=0,455)$ \\
\hline Victimización Física & $\mathrm{M}=1,27(\mathrm{D} \cdot \mathrm{T}=0,344)$ \\
\hline Victimización Verbal & $\mathrm{M}=1,72(\mathrm{D} \cdot \mathrm{T}=0,550)$ \\
\hline
\end{tabular}

Tabla I. Descriptivos de las variables.

Además en el estudio comparativo se aprecia como en referencia a la Residencia Habitual, no se generaron diferencias en función del Género $(\mathrm{p}=.232)$ y Actividad Física $(\mathrm{p}=.556)$, pero si con Bullying, Conducta Violenta y Victimización $\left(\mathrm{p} \leq .005^{* *}\right)$. Específicamente lo adolescentes residentes fuera del contexto familiar eran más acosadores y habían sufrido más acoso que los que vivían en el domicilio familiar, asimismo presentaban puntuaciones incrementadas en índices de agresividad y victimización, como puede constatarse en la siguiente tabla. (Tabla II). 


\begin{tabular}{|c|c|c|c|c|c|c|c|}
\hline \multicolumn{2}{|c|}{ Parámetros } & \multicolumn{2}{|c|}{ Domicilio Familiar } & \multicolumn{2}{|c|}{$\begin{array}{l}\text { No Domicilio } \\
\text { Familiar }\end{array}$} & \multicolumn{2}{|l|}{$X^{2}$} \\
\hline & & $N$ & $\%$ & $N$ & $\%$ & $p$ & \\
\hline \multirow{2}{*}{ Género } & Masculino & 1092 & $52,8 \%$ & 116 & $57,1 \%$ & \multirow{2}{*}{.232} & \\
\hline & Femenino & 978 & $47,2 \%$ & 87 & $42,9 \%$ & & \\
\hline \multirow{2}{*}{$\begin{array}{l}\text { Actividad } \\
\text { Física } \\
\end{array}$} & Si Practica & 1657 & $80,0 \%$ & 166 & $81,8 \%$ & \multirow{2}{*}{.556} & \\
\hline & No Practica & 413 & $20,0 \%$ & 37 & $18,2 \%$ & & \\
\hline \multirow{4}{*}{ Bullying } & $\begin{array}{l}\text { Testigo, no } \\
\text { involucrado }\end{array}$ & 1070 & $51,7 \%$ & 115 & $56,7 \%$ & \multirow{4}{*}{$.000 * *$} & \\
\hline & Acosador & 92 & $4,4 \%$ & 22 & $10,8 \%$ & & \\
\hline & Víctima & 281 & $13,6 \%$ & 34 & $16,7 \%$ & & \\
\hline & $\begin{array}{l}\text { Ninguna de las } \\
\text { anteriores }\end{array}$ & 627 & $30,3 \%$ & 32 & $15,8 \%$ & & \\
\hline & & $M$ & D.T & $M$ & D.T & $p$ & $\mathbf{F}$ \\
\hline \multirow{2}{*}{ Agresividad } & Manifiesta & 1,28 & ,324 & 1,38 & ,401 & $.001 * *$ & 15,025 \\
\hline & Relacional & 1,25 & ,262 & 1,33 & 320 & $.002 * *$ & 13,988 \\
\hline \multirow{3}{*}{ Victimización } & Verbal & 1,70 & ,540 & 1,87 & 619 & $.000 * *$ & 17,486 \\
\hline & Física & 1,25 & ,335 & 1,38 & ,406 & $.000 * *$ & 27,992 \\
\hline & Relacional & 1,52 & ,453 & 1,58 & ,468 & .057 & 3,862 \\
\hline
\end{tabular}

Tabla II. Implicación del lugar de residencia con resto de parámetros.

Analizando la Actividad Física y su práctica, los resultados mostraron diferencias estadísticamente significativas $\left(\mathrm{p} \leq .005^{* *}\right)$ en la relación con casi todas las variables, exceptuando Agresividad Relacional $(\mathrm{p}=.647)$ y Victimización Verbal $(\mathrm{p}=.703)$, de esta manera en la siguiente tabla (Tabla III), observamos como los adolescentes practicantes asiduos de actividad física obtenían cifras más elevadas entre los varones, sujetos con agresividad manifiesta y victimización física, mientras que en los participantes no practicantes estos presentaron valores más elevados en aquellos que sufrían acoso o victimización relacional.

\begin{tabular}{|c|c|c|c|c|c|c|c|}
\hline \multirow{2}{*}{\multicolumn{2}{|c|}{ Parámetros }} & \multicolumn{2}{|c|}{ Si A.F } & \multicolumn{2}{|c|}{ No A.F } & \multicolumn{2}{|l|}{$X^{2}$} \\
\hline & & $N$ & $\%$ & $N$ & $\%$ & $p$ & \\
\hline \multirow{2}{*}{ Género } & Masculino & 1090 & $59,8 \%$ & 118 & $26,2 \%$ & \multirow{2}{*}{$.000 * *$} & \\
\hline & Femenino & 733 & $40,2 \%$ & 332 & $73,8 \%$ & & \\
\hline \multirow{5}{*}{ Bullying } & Testigo, no involucrado & 965 & $52,9 \%$ & 220 & $48,9 \%$ & \multirow{4}{*}{$.002 * *$} & \\
\hline & Acosador & 104 & $5,7 \%$ & 10 & $2,2 \%$ & & \\
\hline & Víctima & 242 & $13,3 \%$ & 73 & $16,2 \%$ & & \\
\hline & \multirow[t]{2}{*}{$\begin{array}{l}\text { Ninguna de las } \\
\text { anteriores }\end{array}$} & 512 & $28,1 \%$ & 147 & $32,7 \%$ & & \\
\hline & & $M$ & D.T & $M$ & D.T & $p$ & $\mathbf{F}$ \\
\hline \multirow{2}{*}{ Agresividad } & Manifiesta & 1,30 & ,341 & 1,26 & ,292 & $.010 * *$ & 6,640 \\
\hline & Relacional & 1,26 & ,268 & 1,27 & 270 & .647 & 0,209 \\
\hline \multirow{3}{*}{ Victimización } & Verbal & 1,72 & ,550 & 1,71 & ,546 & .703 & 0,145 \\
\hline & Física & 1,27 & ,341 & 1,23 & ,354 & $.023 * *$ & 5,146 \\
\hline & Relacional & 1,51 & ,445 & 1,57 & ,491 & $.041 * *$ & 4,737 \\
\hline
\end{tabular}

Tabla III. Distribución de la práctica de Actividad Física con resto de variables. 
Los resultados estadísticos que se desprenden de la variable Bullying en relación con género, agresividad y victimización determinó diferencias estadísticamente significativas en todos los casos $\left(\mathrm{p} \leq .005^{* *}\right)$, revelando que había más acosadores entre los varones y que eran más testigos de estos hechos mientras las chicas lo sufrían más y a la vez percibían menos estos hechos. Los datos nos mostraron que entre los agresores sus puntuaciones eran elevadas con respecto a las otras categorías en Agresividad tanto Manifiesta como Relacional, pero también se detectó que estos percibían la victimización con cifras elevadas con respecto a los que habían sido testigo o los que no; situación parecida a la que se establece entre los que sufrían acoso que presentaron valores muy incrementados en las tres subescalas de Victimización pero también superaban a las otras dos categorías en agresividad.

\begin{tabular}{|c|c|c|c|c|c|c|c|c|c|c|c|}
\hline \multicolumn{2}{|c|}{ Parámetros } & \multicolumn{2}{|c|}{$\begin{array}{l}\text { Testigo, no } \\
\text { involucrado }\end{array}$} & \multicolumn{2}{|c|}{ Acosador } & \multicolumn{2}{|c|}{ Víctima } & \multicolumn{2}{|c|}{$\begin{array}{c}\text { Ninguna de } \\
\text { las } \\
\text { anteriores }\end{array}$} & $X^{2}$ & \\
\hline & & $N$ & $\%$ & $N$ & $\%$ & $N$ & $\%$ & $N$ & $\%$ & $p$ & \\
\hline \multirow[t]{3}{*}{ Género } & Masculino & 660 & $54,6 \%$ & 83 & $6,9 \%$ & 141 & $11,7 \%$ & 324 & $26,8 \%$ & $.000 * *$ & \\
\hline & Femenino & 525 & $49,3 \%$ & 31 & $2,9 \%$ & 174 & $16,3 \%$ & 335 & $31,5 \%$ & & \\
\hline & & $M$ & D.T & $M$ & D.T & $M$ & D.T & M & D.T & $p$ & $\mathbf{F}$ \\
\hline \multirow[t]{2}{*}{ Agresividad } & Manifiesta & 1,30 & ,322 & 1,72 & ,576 & 1,30 & ,295 & 1,20 & ,238 & $.000 * *$ & 86,499 \\
\hline & Relacional & 1,26 & ,261 & 1,48 & ,361 & 1,29 & ,279 & 1,21 & ,234 & $.000 * *$ & 35,867 \\
\hline \multirow[t]{3}{*}{ Victimización } & Verbal & 1,66 & ,459 & 1,83 & ,559 & 2,29 & ,648 & 1,53 & ,456 & $.000 * *$ & 181,067 \\
\hline & Física & 1,23 & ,285 & 1,37 & ,389 & 1,56 & ,479 & 1,17 & ,267 & $.000 * *$ & 116,973 \\
\hline & Relacional & 1,48 & ,397 & 1,61 & ,430 & 1,94 &, 556 & 1,39 &, 381 & $.000 * *$ & 132,742 \\
\hline
\end{tabular}

Tabla IV. Bullying en base al resto de variables.

\section{Discusión}

En este estudio se han analizado la relación existente entre una serie de parámetros de residencia, género y actividad física y comportamientos agresivos, de victimización y Bullying, en primer lugar destacar que nuestro estudio presentó valores homogéneos en cuanto a género, mientras que la mayoría de los participantes residían en un contexto familiar y eran asiduos practicantes de actividad física. Más de la mitad de los adolescentes indicaron que sin ser agresores sí que fueron testigos de casos de bullying sin estar involucrados en ellos, datos que nos confirman que estos comportamientos y conductas están arraigadas en estas poblaciones adolescentes, al hilo de esto se manifestaron Cerezo (1997); Avilés y Monjas (2005) y Cerezo (2009) que en sus estudios hallaron valores superiores al $40 \%$ de involucración en actos similares y añadiendo lo plasmado por Blaya (2010) sobre la violencia entre iguales. En lo que respecta a la conducta violenta se mostraron valores similares entre Agresividad Manifiesta y Relacional, mientras que en el caso de la Victimización la de tipo Verbal, mostró una tendencia media superior que las otras dos variantes, estos datos concuerdan con los reportados en sus estudios por el informe del Defensor del Pueblo (AAVV, 2007), si bien la relacional, pese a ser menos visible que las formas física y verbal, puede llegar a tener consecuencias muy negativas en el ajuste psicosocial del adolescente como las derivadas de la violencia manifiesta (Estévez et al., 2012 y Moreno, Estévez, Murgui y Musitu, 2009). 
Centrándonos en el parámetro de residencia habitual, se apreció como la distribución tanto en género como práctica regular de actividad física fue similar, sin embargo en el caso de Bullying, Conducta Violenta y Victimización los adolescentes residentes fuera del contexto familiar eran más acosadores y habían sufrido más acoso que los que vivían en el domicilio familiar, con mayores índices de agresividad y victimización, esto entendemos que viene producido por lo que numerosos autores llaman factores personales de riesgo condicionantes de la aparición de violencia juvenil (Barudy, 1998; Bemak y Keys, 1999, Cerezo, 2006 y Cerezo, 2009 o FernándezMolina, Del Valle, Fuentes, Bernedo y Bravo, 2011). Entendemos que los adolescentes de internado, acogimiento o residencia vienen condicionados indirectamente por dos elementos uno que es el marco social y otro el entorno familiar. Para entender el primero debemos mencionar a Díaz-Aguado, Martínez y Martín (2004), que consideran que para analizar la problemática es imprescindible el estudio del marco social, porque de este van a derivar numerosos hándicaps que afectan a la actitud y educación; en referencia al contexto familiar Oliveros y Barrientos (2007); Villarreal-González, Sánchez-Sosa, Veiga y Del Moral (2011) coinciden en que existe una relación directa con la violencia escolar, puntualizando que jóvenes que crecen en un ambiente familiar positivo, tienen unas relaciones más positivas con sus iguales e incluso Cohen, Esterkind de Chein, Lacunza, Caballero y Martinengh (2011), afirman que en su estudio sobre adolescentes de contextos de pobreza, estos presentan comportamientos de sensibilidad y de interactuación con sus iguales.

La práctica regular de Actividad Física era más asidua entre los varones, sujetos con agresividad manifiesta y con victimización física, entendemos que esto es producido porque los adolescentes practicantes asiduos del deporte empiezan a competir, perder para ellos es frustrante, se preocupan de demostrar mejor habilidad que los demás, todo esto hace que se establezca una relación entre resultado y agresión como señalan en sus estudios Duda, Olson y Templin (1991) y Duda y Huston (1995), trasladando esa competitividad a su vida social de una manera inconsciente, al hilo de esta situación Gómez (2007), señala que conductas violentas en el contexto deportivo tienen solución y estas vienen determinadas por el alto potencial de autoestima y desarrollo social que genera el deporte en los jóvenes como señala Pelegrín, Martínez y Garcés de los Fayos (2000).

Entre los adolescentes había más acosadores entre los varones e igualmente eran más testigos de hechos violentos mientras las chicas lo sufrían más y a la vez percibían menos estos sucesos, esto confirma lo planteado por Bettencourt y Miller (1996); Olweus (1998); Cerezo (2001); Postigo, González, Mateu, Ferrero y Martorell (2009) y Povedano et al., (2012), que señalan como agresor principalmente al varón (en una proporción de 3 a 1), mientras que las chicas suelen ser víctimas de las agresiones y en ocasiones víctimas-provocadoras, además citan que entre los chicos se implican de una forma muy directa en comportamientos de índole violenta apoyados en la teoría de masculinidad de Connell (1995) y refundando lo señalado por Oliva, Rivera, González y Rey (2012) y mostrando elementos académicos negativos (Torregrosa, Inglés GarcíaFernández, Valle y Núñez, 2012).; asimismo Hilt y Nolen-Hoeksema (2009), hacen mención a que las chicas involucradas en conductas violentas se le implica con bajos niveles autoestima y satisfacción, y en algunos casos con signos de tipo depresivo; y 
Díaz-Aguado et al., (2004), marcan que la exclusión social, es la forma más frecuente de violencia utilizada por ellas.

En general, reflexionamos que este trabajo complementa y aporta nuevas perspectivas al estudio de las conductas violentas de los adolescentes y añade nuevas variables a considerar como es el concepto de alumno interno o residente, asimismo entendemos que entre las limitaciones que presenta el estudio se encuentra la de no incluir subescalas dentro de los parámetros de Actividad Física (si la actividad es individual o colectiva o tipo de actividad) o determinar la regularidad en la práctica, y pone de manifiesto para próximos proyectos la importancia de incluir algún agente de intervención (deporte) como medio de prevención de la agresividad en los adolescentes.

\section{Conclusiones}

Más de la mitad de los adolescentes indicaron que sin ser agresores sí que fueron testigos de casos de Bullying sin estar involucrados en ellos, asimismo los niveles de Agresividad (Manifiesta y Relacional) eran similares, mientras que la Victimización de tipo Verbal, mostró una tendencia media superior a las otras categorías.

Los adolescentes residentes fuera del contexto familiar eran más acosadores y habían sufrido más acoso que los que vivían en el domicilio familiar, obteniendo mayores índices de agresividad y victimización.

La práctica regular de Actividad Física era más asidua entre los varones, y estos se caracterizaban por tener mayor agresividad manifiesta y un índice superior de victimización física.

Había más acosadores entre los varones y estos eran más testigos de hechos violentos mientras las chicas lo sufrían más y a la vez percibían menos situaciones de Bullying.

\section{Referencias bibliográficas}

ABARCA, G.; CARVAJAL, C. y CIFUENTES, A. (2012). Análisis de las concepciones de la masculinidad a la base de la intervención en hombres propuesta por el plan de seguridad pública del Ministerio del Interior. Revista de Psicología, 21(1), 159-184.

ARIAS, W. (2013). Agresión y violencia en la adolescencia: la importancia de la familia. Avances en Psicología, 21(1), 23- 34.

AUSTIN, S. y JOSEPH, S. (1996). Assessment of bully/victim problems in 8 to 11 years old. British Journal of Educational Psychology, 66, 447-456.

AVILÉS, J. M. y MONJAS, I. (2005). Estudio de la incidencia de la intimidación y el maltrato entre iguales en Educación Secundaria Obligatoria mediante el cuestionario CIMEI. Anales de Psicología, 21(1), 27-41. 
BARUDY, J. (1998). El dolor invisible. Una lectura ecosistémica del maltrato infantil. Barcelona: Paidós.

BEMAK, F. y Keys, S. (1999). Violent and agressive youth. Intervention and prevention strategies for changing times. California: Crowing Press.

BETTENCOURT, B. A. y Miller, N. (1996). Gender differences in aggression as a function of provocation: A Meta-analysis. Psychological Bullentin, 119, 422-447.

BLAYA, C. (2010). Violencia y acoso escolar: concepto, incidencia y factores de riesgo y protección. Material Modulo I, Master Oficial en Prevención e intervención psicológica en problemas de conducta. Universitat Internacional Valenciana.

BUELGA, S.; CAVA, M. J. Y MUSITU, G. (2012). Reputación social, ajuste psicosocial y victimización entre adolescentes en el contexto escolar. Anales de Psicología, 28(1), 180-187.

CARROLL, A.; HOUGHTON, S. y BAGLIONI, J.R. (2000). Goals and reputations amongst young children. The validation of the importance of goals and reputation enhancement scales. Social Psychology International, 21, 115-135.

CAVA, M.J.; MUSITU, G. y MURGUI, S. (2006). Familia y violencia escolar: el rol mediador de la autoestima y la actitud hacia la autoridad institucional. Psicothema, 18(3), 367-373.

CAVA, M. J.; MUSITU, G. y MURGUI, S. (2007). Individual and social risk factors related to overt victimization in a sample of Spanish adolescents. Psychological Reports, 101, 275-290.

CEREZO, F. y ATO, M. (2010). Social status, gender, classroom climate and bullying among adolescents pupils. Anales de Psicología, 26, 137-144.

CEREZO, F. (1997). Conductas agresivas en la edad escolar. Madrid: Pirámide.

CEREZO, F. (2001). Variables de personalidad asociadas a la dinámica bullying (agresores versus víctimas) en niños y niñas de 10 a 15 años. Anales de Psicología, 17, 37-44.

CEREZO, F. (2006). Violencia y victimización entre escolares. El bullying: estrategias de identificación y elementos para la intervención a través del test BULL-S. Revista de Investigación Psicoeducativa, 4, 106-114.

CEREZO, F. (2009). Bullying: análisis de la situación en las aulas españolas. International Journal of Psychology and Psychological Therapy. 9(3), 367-378.

CHOE, D. E. y ZIMMERMAN, M. A. (2014). Transactional Process of African American Adolescents' Family Conflict and Violent Behavior. Journal of Research Adolescence, 24(4), 591-597.

COHEN, S.; ESTERKIND DE CHEIN, A. E.; LACUNZA, A. B.; CABALLERO, S. V. y MARTINENGH, C. (2011). Habilidades y contexto sociocultural. Un estudio con adolescentes a través del BAS-3. Revista Iberoamericana de Diagnóstico y 
Evaluación Psicológica, 29(1), 167-185.

CONNELL, R. W. (1995). Masculinities: knowledge, power and social change. Cambridge: Polity Press.

COOK, C.; WILLIAMS, K.; GUERRA, N.; KIM, T. y SADEK, S. (2010). Predictors of Bullying and Victimization in Childhood and Adolescence: A Meta-analytic Investigation. School Psychology Quarterly, 25(2), 65- 83.

DEFENSOR DEL PUEBLO (2000). Violencia escolar: El maltrato entre iguales en la Educación Secundaria Obligatoria. Informe de la Oficina del Defensor del Pueblo. Madrid

DEFENSOR Del PUEBLO (2007). Violencia escolar: El maltrato entre iguales en la Educación Secundaria Obligatoria 1999-2006 (Nuevo estudio y actualización del informe 2000). Informe de la Oficina del Defensor del Pueblo. Madrid.DíazAguado, M.J.; Martínez, R. y Martín, G. (2004). La violencia entre iguales en la escuela y en el ocio. Estudios comparativos e instrumentos de evaluación. Madrid: Instituto de la Juventud.

DUDA, J.L.; OLSON, L. K. y TEMPLIN, T. J. (1991). The relation of task and ego orientations to sportsmanship attitudes and the perceived legitimacy of injurius acts. Research Quaterly for Exercise and Sport. 62, 79-87

DUDA, J.L. y HUSTON, L. (1995).The relationship of goal orientation and degree of competitive sport participation to the endorsement of aggressive acts in American football. IXth Europen Congresson Sport Psychology, 665-662.

ESTÉVEZ, E. (2005). Violencia, Victimización y Rechazo Escolar en la Adolescencia. Universitat de Valencia: Servei de publicacions.

ESTÉVEZ, E.; INGLÉS, C.; EMLER, N.; MARTÍNEZ-MONTEAGUDO, M. C. y Torregrosa, M. S. (2012). Análisis de la relación entre la victimización y la violencia escolar: El rol de la reputación antisocial. Intervención Psicosocial, 21, 53-65.

FERNÁNDEZ-MOLINA M.; DEL VALLE J.; FUENTES M. J.; BERNEDO I. y BRAVO A. (2011). Problemas de conducta de los adolescentes en acogimiento preadoptivo, residencial y con familia extensa. Psicothema, 23(1), 1-6.Gómez, A. (2007). La violencia en el deporte: un análisis desde la psicología social. Revista de Psicología Social, 22(1), 63-87.

GONZÁLEZ-JIMÉNEZ, R. M. (2009). Presentación. Estudios de género en Educación: una rápida mirada. Revista Mexicana de Investigación Educativa, 14(42), 681-699.

GUERRA, C.; ÁLVAREZ-GARCÍA, D.; DOBARRO, A.; NÚÑEZ, J. C.; CASTRO, L. y VARGAS, J. (2011). Violencia escolar en estudiantes de Educación Secundaria de Valparaíso (Chile): Comparación con una muestra española. Revista Iberoamericana de Psicología y Salud, 2, 75-98.

HAYNIE, D. L.; DOOGAN, N. J. y SOLLER, B. (2014). Gender, friendship networks, 
and delinquency: A dinamic network aproach. Criminology, 52(4), 688-722.

HILT, L. y NOLEN-HOEKSEMA, S. (2009). The emergence of gender differences in depression in adolescence. En S. Hoeksema (Ed.), Handbook of depression in adolescents (pp. 111-135). Nueva York: Routledge.

JIMÉNEZ, T. I.; MORENO, D.; MURGUI, S. y MUSITU, G. (2008). Factores psicosociales relacionados con el estatus social del alumno en el aula: El rol de la reputación social, la amistad, la conducta violenta y la relación con el profesor. International Journal of Psychology and Psychologica Therapy, 8, 227-236.

JIMÉNEZ, T. I.; MUSITU, G.; RAMOS, M. J. y MURGUI, S. (2009). Community involvement and victimization at school: an analysis through family, personal and social adjustment. The Journal of Community Psychology, 37(8), 959-974.

LAZO, H.E. y SALAZAR, M.M. (2011). Bullying "destruyendo mi autoestima". Revista Salud, Sexualidad y Sociedad, 3(4), 1-4.

LiTTle, T. D.; HENRICH, C. C.; JONES, S. M. y HAWLEY, P. H. (2003). Disentangling the "whys" from the "whats" of aggressive behaviour. International Journal of Behavioral Development, 27, 122-133.

LÓPEZ-ROMERO, L. y ROMERO, E. (2010). Goals during adolescence and their relationship with antisocial behavior. The Spanish Journal of Psychology, 13, 166177.

MARTORELL, C.; GONZÁLEZ, R.; ORDOÑEZ, A. y GÓMEZ, O. (2011). Estudio confirmatorio del cuestionario de conducta antisocial (CCA) y su relación con variables de personalidad y conducta antisocial. Revista Iberoamericana de Diagnóstico y Evaluación Psicológica, 31(1), 97-114.

MORENO, D.; ESTÉVEZ, E.; MURGUI, S. y MUSITU, G. (2009). Reputación social y violencia relacional en adolescentes: el rol de la soledad, la autoestima y la satisfacción vital. Psicothema, 21, 537-542.

MUSITU, G.; ESTÉVEZ, E. y EMLER, N. (2007). Adjustment problems in the family and school contexts, attitude towards authority and violent behaviour at school in adolescence. Adolescence, 42, 779-794.

MYNARD, H. y JOSEPH, S. (2000). Development of the Multidimensional PeerVictimization Scale. Aggressive Behavior, 26, 169-178.

OLIVA, L.; RIVERA, E. A.; GONZÁLEZ, M. P. y REY, L. (2012). Comportamientos agresivos en estudiantes de 4 hasta 22 años de Xalapa- México. Revista Iberoamericana para la Investigación y el Desarrollo Educativo, 9, 1-18.

OLIVEROS, M. y BARRIENTOS, A. (2007). Incidencias y factores de riesgo de la intimidación (bullying) en un colegio particular de Lima-Perú. Revista Peruana de Pediatría, 60(3), 150-155.

OLWEUS, D. (1978). Aggression in the schools: Bullies and whipping boys. Washington, D.C.: Hemisphere. 
OLWEUS, D. (1998). Conductas de acoso y amenaza entre escolares. Madrid: Morata. OPPENHEIMER, O. (2008). Cuentos chinos. Buenos Aires: Editorial Sudamericana.

ORTEGA, R.; CALMAESTRA, J. y MORA, J. (2008). Ciberbullying. Internacional Journal of Psychology and Psychotherapy, 8, 183-192.

PABAYO, R.; MOLNAR, B. E. y KAWACHI, I. (2014). The role of Neighborhood Income Inequality in Adolescent Aggression and Violence. Journal of Adolescent Health, 55(4), 571-579.

PECHORRO, P., GONCALVES, R. A., MAROCO, J., GAMA, A. P., NEVES, S. Y NUNES, C. (2014). Juvenile delinquency and psychopathic traits: An empirical study with Portuguese adolescents. International Journal of Offender Therapy and Comparative Criminology, 58(2), 174-189.

PEDERSON, S.; SEIDMAN, E.; YOSHIKAWA, H.; RIVERA, A. y ALLEN, L, (2005). Contextual competence: Multiple manifestations among urban adolescents. American Journal Community Psychology, 35, 65-82.

PELEGRÍN, A.; MARTÍNEZ, F. y GARCÉS DE LOS FAYOS E.J. (2000). La actividad física y el deporte como factores controladores de conductas antisociales y delictivas. II Congreso Iberoamericano de Psicología del Deporte. Huelva.

POSTIGO, S.; GONZÁleZ, R.; MATEU, C.; FERRERO, J. y MARTORELL, C. (2009). Diferencias conductuales según género en convivencia escolar. Psicothema, 21, 453-458.

POVEDANO, A.; ESTÉVEZ, E.; MARTÍNEZ, B. y MONREAL, M. C. (2012). Un perfil psicosocial de adolescentes agresores y víctimas en la escuela: análisis de las diferencias de género. Revista de Psicología Social, 27(2), 169-182.

RENOLD, E. (2004). Other boys: negotiating non-hegemonic masculinities in the primary school. Gender and Education, 16(2), 247-266.

RODRÍGUEZ, R.; SEOANE, A. y PEDREIRA, J.L. (2006). Niños contra niños: el bullying como trastorno emergente. Anales de pediatría, 64 (2), 162-166.

SANTOS, J.; MUÑOZ, P.; JUEZ, J. y CORTIÑAS, A. (2003). Diseño de Encuestas para estudios de mercado. Técnicas de muestreo y análisis multivariante. Madrid: Editorial Ramón Areces.

SCHWARTZ, D. (2000). Subtypes of victims and aggressors in children's peer groups. Journal of Abnormal Child Psychology, 28, 181-192.

SCOTT, J. W. (1992). Igualdad versus diferencia: los usos de la teoría posestructuralista. Debate Feminista, 3(5), 85-104.

SMITH, P. K. (2003). Violence in schools: The response in Europe. Londres: Routledge Falmer.

TORREGROSA, M., S.; INGLÉS C. J.; GARCÍA-FERNÁNDEZ, J. M.; VALLE, A. y NÚÑEZ J. C. (2012). Relaciones entre conducta agresiva y metas académicas: 
estudio con una muestra de estudiantes españoles de Educación Secundaria Obligatoria. Universitas Psychologica, 11(4), 1303-1315.

TRICKETT, E. J. (2009). Community psychology: Individuals and interventions in community context. Annual Review of Psychology, 60, 395-419.

VILLARREAL-GONZÁLEZ, M.E.; SÁNCHEZ-SOUSA, J.C.; VEIGA, F. y DEL MORAL, G. (2011). Contextos de desarrollo, malestar psicológico, autoestima social y violencia escolar desde una perspectiva de género en adolescentes mexicanos. Psychosocial Intervention, 20(2), 171-181.

YUBERO, S.; OVEJERO, A. y LARRAÑAGA, E. (2010). Apoyo social percibido en el contexto escolar y victimización entre iguales durante la adolescencia. Revista de Psicología Social, 25, 283-293.

ZEIGLER-HILL, V., ENJAIAN, B., HOLDEN, C. J. Y SOUTHARD, A. C. (2014). Using self-steem inestability to disentrangle the connection between self-steem level and perceived aggression. Journal of Research in Personality, 49, 47-51.

\section{Correspondencia con autores}

Félix ZURITA ORTEGA

Facultad de Ciencias de la Educación (Despacho 122.1)

Campus de Cartuja s/n

18071 Granada

e.mail: felixzo@ugr.es 